University of Wollongong

Research Online

Faculty of Informatics - Papers (Archive)

Faculty of Engineering and Information

Sciences

2008

\title{
Hierarchical Identity-Based Online/Offline Encryption
}

Zhongren Liu

Fujian Normal University

Li Xu

Fujian Normal University

Zhide Chen

Fujian Normal University

Yi Mu

University of Wollongong, ymu@uow.edu.au

Fuchun Guo

Fujian Normal University, fuchun@uow.edu.au

Follow this and additional works at: https://ro.uow.edu.au/infopapers

Part of the Physical Sciences and Mathematics Commons

\section{Recommended Citation}

Liu, Zhongren; Xu, Li; Chen, Zhide; Mu, Yi; and Guo, Fuchun: Hierarchical Identity-Based Online/Offline Encryption 2008.

https://ro.uow.edu.au/infopapers/3191

Research Online is the open access institutional repository for the University of Wollongong. For further information contact the UOW Library: research-pubs@uow.edu.au 


\title{
Hierarchical Identity-Based Online/Offline Encryption
}

\author{
Abstract \\ The notion of identity-based online/offline encryption (IBOOE) was recently introduced by Guo, Mu and \\ Chen in FC 2008. In an IBOOE system, the encryption is split into online and offline phases. The offline \\ phase is performed prior to the arrival of a message and the recipient's public key (or,identity). The online \\ phase is performed very efficiently after knowing the message and public key. The IBOOE scheme is \\ particularly useful for devices that have very low computation power since partof computation is \\ conducted while the device is not busy. In this paper, we extend the notion of IBOOE to the hierarchical \\ identity-based online/offline encryption (HIBOOE), and propose a "selective-ID" secure HIBOOE scheme \\ from Boneh, Boyen and Goh's HIBE, where the online phase in HIBOOE is very efficient.

\section{Disciplines} \\ Physical Sciences and Mathematics \\ Publication Details \\ Liu, Z., Xu, L., Chen, Z., Mu, Y. \& Guo, F. (2008). Hierarchical Identity-Based Online/Offline Encryption. In G. \\ Wang, J. Chen, M. Fellows \& H. Ma (Eds.), International Conference for Young Computer Scientists \\ (ICYCS) 2008 (pp. 2115-2119). USA: IEEE.
}




\title{
Hierarchical Identity-Based Online/Offline Encryption
}

\author{
Zhongren $\mathrm{Liu}^{1}, \mathrm{Li} \mathrm{Xu}^{1}$, Zhide $\mathrm{Chen}^{1}$, Yi Mu${ }^{2}$ and Fuchun Guo ${ }^{1}$ \\ ${ }^{1}$ School of Mathematics and Computer Science \\ Fujian Normal University, Fuzhou, China \\ zhongren88@163.com \\ ${ }^{2}$ School of Computer Science and Software Engineering \\ University of Wollongong, Wollongong NSW 2522, Australia \\ ymu@uow.edu.au
}

\begin{abstract}
The notion of Identity-Based Online/Offline Encryption (IBOOE) was recently introduced by Guo, Mu and Chen in FC 2008. In an IBOOE system, the encryption is split into online and offline phases. The offline phase is performed prior to the arrival of a message and the recipient's public key (or, identity). The online phase is performed very efficiently after knowing the message and public key. The IBOOE scheme is particularly useful for devices that have very low computation power since part of computation is conducted while the device is not busy. In this paper, we extend the notion of IBOOE to the Hierarchical IdentityBased Online/Offline Encryption (HIBOOE), and propose a "selective-ID" secure HIBOOE scheme from Boneh, Boyen and Goh's HIBE, where the online phase in HIBOOE is very efficient.
\end{abstract}

Keywords: Identity based, encryption, online, offline.

\section{Introduction}

Identity Based Encryption (IBE) is a public key cryptsystem where any arbitrary string such as an email address or a telephone number can be utilized as a valid public key. The corresponding user private keys can only be computed by a trusted third party called the Private Key Generator (PKG) (who possesses a master secret key). The notion of identity based cryptography was first proposed by Shamir in 1984 [10]. This notion was later extended to IBE (e.g., $[1,3,6,12])$. In a traditional IBE scheme, there is only one PKG that distributes private keys for users. To improve the efficiency of key generation, Horwitz and Lynn first introduced the notion of Hierarchical Identity-Based Encryption (HIBE) in [9]. HIBE is a generalization of IBE that mirrors an organizational hierarchy. In an HIBE system, there is a root PKG who has a master secret key, some domain PKGs, and users. The domain PKGs and users are all associated with their ID which are arbitrary strings. Root PKG generates a private key for the top-level domain PKG. The lower-level PKG requests a private key form their parent domain PKGs. Users ask for their private keys from their domain PKGs. It is noticed that several efficient HIBE schemes were proposed in $[1,2,8,11]$ with or without random oracles.

Recently, Guo, Mu and Chen [7] introduced the notion of identity-based online/offline encryption (IBOOE). The basic concept of online/offline encryption lies in splitting the encryption algorithm into two phases, the first phase is performed offline prior to the arrival of a message to be encrypted and a public key (identity ID). The second phase is performed online after knowing the message and ID. The online phase is typically very fast and the offline phase is designed to handle the most costly computation. This scheme is particularly useful for weak devices that do not have sufficient computation capacity.

In this paper, we extend the IBOOE to the Hierarchical Identity-Based Online/Offline Encryption (HIBOOE) and describe how to construct an HIBOOE scheme where the public key is a multi-tuple vector of domain identities. Although the IBOOE [7] scheme from Boneh-Boyen IBE can be extended to HIBOOE in a trivial way, the construction unfortunately results in a longer ciphertext and multiple modular computations in the online phase. In this paper, we construct a much more efficient HIBOOE from another HIBE [2], which was proposed by Boneh, Boyen and Goh in 2005 to construct the constant ciphertext of HIBE. Our HIBOOE scheme performs very efficiently in the online phase which requires two modular computations only and has a shorter ciphertext with $k+4$ elements (where $k$ is the 
length of the public key), compared to $k+1$ modular computations and $2 k+3$ elements of ciphertext length from the Bonen-Boyen HIBE. Different to the HIBOOE construction from the Boneh-Boyen HIBE scheme, the original security proof for [2] is no longer adapted to our HIBOOE scheme. Therefore, we give a new security proof in the selective identity model in order to support our construction.

The organization of the paper is as follows. In Section 2 , we provide the definitions of IBE/HIBE, including security requirements. In Sections 3, we present our HIBOOE scheme from the Boneh, Boyen and Goh's HIBE (BBGHIBE) scheme. We give a comparison in Section 4 and conclude our paper in Section 5.

\section{Preliminaries}

\subsection{Security models}

An IBE system is composed of the four randomized algorithms: Setup, KeyGen, Encrypt, and Decrypt for master params and master secret key generation, private key generation, encryption and decryption, respectively. An $\ell$-HIBE has a family of $\ell$ key-generation algorithms (KeyGen Kor f $i \leq \ell$ ). The public keys now forms a multi-tuple vector. Algorithm $\operatorname{KeyGen}_{i}$ takes as input an identity $I D=\left(I_{1}, \cdots, I_{i}\right)$ at depth $i$ and the private key $d_{I D \mid i-1}$ of the parent identity $I D_{i-1}=\left(I_{1}, \cdots, I_{i-1}\right)$ at depth $i-1$, then outputs the private key $d_{I D}$ for identity $I D$.

\subsubsection{IND-sID-CCA Model.}

We describe the indistinguishable selective-identity chosenciphertext attack model as follows:

Initialization: The adversary outputs an identity $I D^{*}$ to be challenged.

Setup: The challenger inputs a security parameter $1^{k}$, and then runs the algorithm Setup. It gives the adversary the resulting master public parameters denoted by params and keeps the master secret key to itself.

Phase 1: The adversary makes queries $q_{1}, q_{2}, \cdots, q_{m}$, where $q_{i}$ is one of the following:

- Key generation query on $\left\langle I D_{i}\right\rangle$ where $I D_{i} \neq I D^{*}$ and $I D_{i}$ is not a prefix of $I D^{*}$. The challenger responds by running algorithm KeyGen to generate the private key $d_{I D_{i}}$ and sends it to the adversary.

- Decryption query $\left\langle I D_{i}, C_{i}\right\rangle$. The challenger responds by running algorithm KeyGen to generate the private key $d$ corresponding to $I D_{i}$, running algorithm Decrypt to decrypt the ciphertext $C_{i}$ using the private key $d$ and sending the result to the adversary.
These queries may be asked adaptively according to the replies of queries.

Challenge: Once the adversary decides that Phase 1 is over, it outputs two equal length plaintexts $M_{0}, M_{1}$ on which it wishes to be challenged. The challenger picks a random bit $b \in\{0,1\}$ and sets $C=$ Encrypt $\left(\right.$ params $\left., I D^{*}, M_{b}\right)$. It sends $C$ as the challenge to the adversary.

Phase 2: It is the same as Phase 1 but with a constraint that the adversary makes a decryption query on $\left\langle C_{i}\right\rangle \neq\langle C\rangle$ for $I D^{*}$ or any prefix of $I D^{*}$.

Guess: The adversary outputs a guess $b^{\prime} \in\{0,1\}$ and wins the game if $b^{\prime}=b$.

We refer to such an adversary $\mathcal{A}$ as an IND-sID-CCA adversary. We define the advantage of adversary $\mathcal{A}$ in attacking the scheme $\mathcal{E}$ as

$$
A d v_{\mathcal{E}, \mathcal{A}}=\left|\operatorname{Pr}\left[b^{\prime}=b\right]-\frac{1}{2}\right| .
$$

Definition 1 [1] We say that an HIBE system $\mathcal{E}$ is $\left(t, q_{I D}, q_{c}, \epsilon\right)$-adaptively chosen ciphertext secure if for any t-time IND-sID-CCA adversary $\mathcal{A}$ making at most $q_{I D}$ chosen private key queries and at most $q_{c}$ chosen decryption queries has advantage at most $\epsilon$. As shorthand, we say that $\mathcal{E}$ is $\left(t, q_{I D}, q_{c}, \epsilon\right)$ IND-sID-CCA secure.

Definition 2 [1] We say that an HIBE system $\mathcal{E}$ is $\left(t, q_{I D}, \epsilon\right)$-adaptively chosen plaintext secure if $\mathcal{E}$ is $\left(t, q_{I D}, 0, \epsilon\right)$ adaptively chosen ciphertext secure. As shorthand, we say that $\mathcal{E}$ is $\left(t, q_{I D}, \epsilon\right) I N D$-sID-CPA secure.

\subsection{Bilinear Map}

Let $\mathbb{G}$ and $\mathbb{G}_{1}$ be two cyclic groups of prime order $p$. Let $g$ be a generator of $\mathbb{G}$. A map $e: \mathbb{G} \times \mathbb{G} \rightarrow \mathbb{G}_{1}$ is called a bilinear map if this map satisfies the following properties:

- Bilinear: for all $u, v \in \mathbb{G}$ and $a, b \in \mathbb{Z}_{p}$, we have $e\left(u^{a}, v^{b}\right)=e(u, v)^{a b}$.

- Non-degeneracy: $e(g, g) \neq 1$.

- Computability: It is efficient to compute $e(u, v)$ for all $u, v \in \mathbb{G}$.

\subsection{Complexity Assumption}

We briefly review the $\ell$-Decisional Bilinear DiffieHellamn Inversion $(\ell$-DBDHI) problem and $\ell$-Weak Decisional Bilinear Diffie-Hellamn Inversion $(\ell-w D B D H I)$ problem [1, 2]. 
Definition 3 Let $\mathbb{G}$ and $\mathbb{G}_{1}$ be two multiplicative groups of prime order $p$. Let $w \in \mathbb{G}$ be a generator and $\beta \in$ $\mathbb{Z}_{p}^{*}$. Given elements $w, w^{\beta}, w^{\left(\beta^{2}\right)}, \cdots, w^{\left(\beta^{\ell}\right)} \in \mathbb{G}$, the $\ell$ DBDHI problem in $\left(\mathbb{G}, \mathbb{G}_{1}\right)$ is to decide whether a random value $Z \in \mathbb{G}_{1}$ is equal to $e(w, w)^{1 / \beta}$ or not.

Definition 4 We say that the $(t, \epsilon, \ell)-D B D H I$ assumption holds in $\left(\mathbb{G}, \mathbb{G}_{1}\right)$ if no t-time algorithm has advantage at least $\epsilon$ in solving the $\ell-D B D H I$ problem in $\left(\mathbb{G}, \mathbb{G}_{1}\right)$.

Definition 5 Let $\mathbb{G}$ and $\mathbb{G}_{1}$ be two multiplicative groups of prime order $p$. Let $g, h \in \mathbb{G}$ be two generators and $\alpha \in \mathbb{Z}_{p}^{*}$.

(1) Given elements $g, h, g^{\alpha}, g^{\left(\alpha^{2}\right)}, \cdots, g^{\left(\alpha^{\ell}\right)} \in \mathbb{G}$, the $\ell$ wDBDHI problem in $\left(\mathbb{G}, \mathbb{G}_{1}\right)$ is to decide whether a random value $Z \in \mathbb{G}_{1}$ is equal to $e(g, h)^{\left(\alpha^{\ell+1}\right)}$ or not.

(2) Given elements $g, h, g^{\alpha}, g^{\left(\alpha^{2}\right)}, \cdots, g^{\left(\alpha^{\ell}\right)}, g^{\left(\alpha^{\ell+2}\right)}, \cdots$ ,$g^{\left(\alpha^{2 \ell}\right)} \in \mathbb{G}$, the $\ell-w D B D H I^{*}$ problem in $\left(\mathbb{G}, \mathbb{G}_{1}\right)$ is to decide whether a random value $Z \in \mathbb{G}_{1}$ is equal to $e(g, h)^{\left(\alpha^{\ell+1}\right)}$ or not.

Definition 6 We say that the $(t, \epsilon, \ell)$-wDBDHI (*) assumption holds in $\left(\mathbb{G}, \mathbb{G}_{1}\right)$ if no t-time algorithm has advantage at least $\epsilon$ in solving the $\ell-w D B D H I(*)$ problem in $\left(\mathbb{G}, \mathbb{G}_{1}\right)$.

Definition 7 Let $y_{i}=g^{\left(\alpha^{i}\right)} \in \mathbb{G}^{*}$ and $\vec{y}_{g, a, \ell}=$ $\left(y_{1}, \cdots, y_{\ell}, y_{\ell+2}, \cdots, y_{2 \ell}\right)$. An algorithm $\mathcal{B}$ has advantage $\epsilon$ in solving $\ell-w D B D H I^{*}$ in $\mathbb{G}$ if

$$
\begin{aligned}
& \mid \operatorname{Pr}\left[\mathcal{B}\left(g, h, \vec{y}_{g, a, \ell}, e(g, h)^{a^{\ell+1}}\right)=0\right] \\
& \quad-\operatorname{Pr}\left[\mathcal{B}\left(g, h, \vec{y}_{g, a, \ell}, T\right)=0\right] \mid \geq \epsilon,
\end{aligned}
$$

where the probability is over the random choice of generators $g, h$ in $\mathbb{G}^{*}$, the random choice of $\alpha$ in $\mathbb{Z}_{p}^{*}$, the random choice of $T \in \mathbb{G}_{1}^{*}$, and the random bits consumed by $\mathcal{B}$. We refer to the distribution on the left as $\mathcal{P}_{w B D H I^{*}}$ and the distribution on the right as $\mathcal{R}_{w B D H I^{*}}$.

\section{HIBOOE from BBG-HIBE}

\subsection{Construction}

Let $\mathbb{G}$ be a bilinear group of prime order $p$ and let $e$ : $\mathbb{G} \times \mathbb{G} \rightarrow \mathbb{G}_{1}$ be a bilinear map. We assume that public keys (that is, identities $I D$ ) at level $k$ are vectors of elements in $\left(\mathbb{Z}_{p}^{*}\right)^{k}$. We write $I D=\left(I_{1}, \cdots, I_{k}\right) \in\left(\mathbb{Z}_{p}^{*}\right)^{k}$. The $j$-th component corresponds to the identity at level $j$. If necessary, we extend the construction to public keys over $\{0,1\}^{*}$ by first hashing some components $\left\{I_{j} \mid 1 \leq j \leq k\right\}$ using a collision resistant hash $H:\{0,1\}^{*} \rightarrow \mathbb{Z}_{p}^{*}$. We also assume that the messages to be encrypted are elements in $\mathbb{G}_{1}$. The HIBE system works as follows:
Setup: To generate system parameters for an HIBE of maximum level $\ell$, select a random generator $g$ in $\mathbb{G}^{*}$, a random $a \in \mathbb{Z}_{p}$, and set $g_{1}=g^{a}$. Next, pick random elements $g_{2}, g_{3}, h_{1}, \cdots, h_{\ell} \in \mathbb{G}$. The public parameters and secret master-key are

$$
\begin{aligned}
& \text { params }=\left(g, g_{1}, g_{2}, g_{3}, h_{1}, \cdots, h_{\ell}\right), \\
& \text { master-key }=g_{2}^{a} .
\end{aligned}
$$

$\operatorname{KeyGen}\left(d_{I D}, I D\right)$ : To generate a private key $d_{I D}$ for an identity $I D=\left(I_{1}, \cdots, I_{k}\right) \in\left(\mathbb{Z}_{p}^{*}\right)^{k}$ of depth $k \leq \ell$, using the master secret, pick a random $r \in \mathbb{Z}_{p}$ and output

$$
\begin{aligned}
d_{I D} & =\left(g_{2}^{a} \cdot\left(h_{1}^{I_{1}} \cdots h_{k}^{I_{k}} \cdot g_{3}\right)^{r}, g^{r}, h_{k+1}^{r}, \cdots, h_{\ell}^{r}\right) \\
& =\left(a_{0}, a_{1}, b_{k+1}, \cdots, b_{\ell}\right) \in \mathbb{G}^{2+\ell-k}
\end{aligned}
$$

Note that $d_{I D}$ becomes shorter as the depth of $I D$ increases. The private key for $I D$ can be generated incrementally, given a private key for the parent identity $I D_{k-1}=\left(I_{1}, \cdots, I_{k-1}\right) \in\left(\mathbb{Z}_{p}^{*}\right)^{k-1}$, as required. Indeed, let

$$
\begin{aligned}
d_{I D \mid k-1} & =\left(g_{2}^{a} \cdot\left(h_{1}^{I_{1}} \cdots h_{k-1}^{I_{k-1}} \cdot g_{3}\right)^{r^{\prime}}, g^{r^{\prime}}, h_{k}^{r^{\prime}}, \cdots, h_{\ell}^{r^{\prime}}\right) \\
& =\left(a_{0}^{\prime}, a_{1}^{\prime}, b_{k}^{\prime}, \cdots, b_{\ell}^{\prime}\right)
\end{aligned}
$$

be the private key for $I D_{k-1}$. To generate $d_{I D}$, pick a random $t \in \mathbb{Z}_{p}$ and output

$$
\begin{aligned}
d_{I D}=\left(a_{0}^{\prime} \cdot b_{k}^{I_{k}} \cdot\left(h_{1}^{I_{1}} \cdots h_{k}^{I_{k}} \cdot g_{3}\right)^{t}, a_{1}^{\prime} g^{t} s,\right. \\
\left.b_{k+1}^{\prime} h_{k+1}^{t}, \cdots, b_{\ell}^{\prime} h_{\ell}^{t}\right) .
\end{aligned}
$$

The private key is a properly distributed private key for $I D=\left(I_{1}, \cdots, I_{k}\right)$ for $r=r^{\prime}+t \in \mathbb{Z}_{p}$.

Encrypt(params, $I D, M)$ : We refer to the original BBGHIBE as general encryption. It is not required in our HIBOOE, but since our HIBOOE decryption is associated with the BBG-HIBE, we outline the scheme as follows:

General Encryption: To encrypt a message $M \in \mathbb{G}_{1}$ under the public key $I D=\left(I_{1}, \cdots, I_{k}\right) \in\left(\mathbb{Z}_{p}^{*}\right)^{k}$, pick a random $s \in \mathbb{Z}_{p}$ and output

$$
\begin{aligned}
C_{\mu} & =\left(e\left(g_{1}, g_{2}\right)^{s} \cdot M, g^{s},\left(h_{1}^{I_{1}} \cdots h_{k}^{I_{k}} \cdot g_{3}\right)^{s}\right) \\
& =\left(c_{0}, c_{1}, c_{2}\right) .
\end{aligned}
$$

Online/Offline Encryption: We now describe our HI- 
BOOE, which is divided into two phases:

- Offline Encryption: Choose random $s, \beta, \alpha_{1}, \cdots, \alpha_{\ell}$ $\in \mathbb{Z}_{p}$, and output

$$
\begin{aligned}
& C_{o f} \\
= & \left(e\left(g_{1}, g_{2}\right)^{s},\left(g_{3}^{\alpha_{1}} h_{1}\right)^{s}, \cdots,\left(g_{3}^{\alpha_{\ell}} h_{\ell}\right)^{s},\left(g_{3}^{\beta}\right)^{s}, g^{s}\right) \\
= & \left(c_{0}^{\prime}, c_{1}, \cdots, c_{\ell}, \nu_{1}, \nu_{2}\right) .
\end{aligned}
$$

Store the offline parameters $C_{o f}, \beta^{-1}, \alpha_{1}, \cdots, \alpha_{\ell}$ for the online phase.

- Online Encryption: Given a message $M \in \mathbb{G}_{1}$ and the public key $I D=\left(I_{1}, \cdots, I_{k}\right) \in\left(\mathbb{Z}_{p}^{*}\right)^{k}$, and output

$$
C_{\text {on }}=\left(c_{0}^{\prime} \cdot M, \beta^{-1}\left(1-\sum_{i=1}^{k} \alpha_{i} I_{i}\right)\right)=\left(c_{0}, \nu_{3}\right) .
$$

The ciphertext for $I D$ is $C=\left(c_{0}, c_{1}, \cdots, c_{k}, \nu_{1}, \nu_{2}\right.$ ,$\left.\nu_{3}\right)$, where

$$
\begin{array}{r}
C=\left(e\left(g_{1}, g_{2}\right)^{s} \cdot M,\left(g_{3}^{\alpha_{1}} h_{1}\right)^{s}, \cdots,\left(g_{3}^{\alpha_{k}} h_{k}\right)^{s},\right. \\
\left.\left(g_{3}^{\beta}\right)^{s}, g^{s}, \beta^{-1}\left(1-\sum_{i=1}^{k} \alpha_{i} I_{i}\right)\right) .
\end{array}
$$

Observe that the online phase has a very low computational complexity and the offline phase dose not require the knowledge of the message and the public key (ID) of a recipient. The length of ciphertext is $k+4(k \leq \ell)$, which is acceptable since the parameter $\ell$ is limited.

$\operatorname{Dencrypt}\left(d_{I D}, C\right)$ : We describe our HIBOOE decryption and general decryption in this phase.

HIBOOE Dencryption: Let $C=\left(c_{0}, c_{1}, \cdots, c_{k}, \nu_{1}\right.$, $\left.\nu_{2}, \nu_{3}\right)$ to be a valid ciphertext for $I D=$ $\left(I_{1}, \cdots, I_{k}\right) \in\left(\mathbb{Z}_{p}^{*}\right)^{k}$. To decrypt $C$ with $d_{I D}$, compute

$$
\begin{aligned}
c= & \left(c_{1}\right)^{I_{1}} \cdots\left(c_{k}\right)^{I_{k}} \cdot\left(\nu_{1}\right)^{\nu_{3}} \\
= & \left(\left(g_{3}^{\alpha_{1}} h_{1}\right)^{s}\right)^{I_{1}} \cdots\left(\left(g_{3}^{\alpha_{k}} h_{k}\right)^{s}\right)^{I_{k}} \cdot \\
& \left(\left(g_{3}^{\beta}\right)^{s}\right)^{\beta^{-1}\left(1-\sum_{i=1}^{k} \alpha_{i} I_{i}\right)} \\
= & \left(g_{3}^{\sum_{i=1}^{k} \alpha_{i} I_{i}} h_{1}^{I_{1}} \cdots h_{k}^{I_{k}}\right)^{s} \cdot g_{3}^{s\left(1-\sum_{i=1}^{k} \alpha_{i} I_{i}\right)} \\
= & \left(h_{1}^{I_{1}} \cdots h_{k}^{I_{k}} \cdot g_{3}\right)^{s} .
\end{aligned}
$$

We then have $\left(c_{0}, \nu_{2}, c\right)=\left(e\left(g_{1}, g_{2}\right)^{s} \cdot M, g^{s}\right.$, $\left.\left(h_{1}^{I_{1}} \cdots h_{k}^{I_{k}} \cdot g_{3}\right)^{s}\right)$, which is the same as the output of the general encryption described earlier in this section and the message can be recovered with the general decryption procedure as below.

General Decryption: We refer to the decryption process of the original BBG-HIBE as general decryption. Consider an identity $I D=\left(I_{1}, \cdots, I_{k}\right)$. To decrypt a given ciphertext $C_{\mu}=\left(c_{0}, c_{1}, c_{2}\right)$ using the private key $d_{I D}=\left(a_{0}, a_{1}, b_{k+1}, \cdots, b_{\ell}\right)$, output

$$
c_{0} \cdot e\left(a_{1}, c_{2}\right) / e\left(c_{1}, a_{0}\right)=M
$$

Indeed, for a valid ciphertext, we have

$$
\begin{aligned}
\frac{e\left(a_{1}, c_{2}\right)}{e\left(c_{1}, a_{0}\right)} & =\frac{e\left(g^{r},\left(h_{1}^{I_{1}} \cdots h_{k}^{I_{k}} \cdot g_{3}\right)^{s}\right)}{e\left(g^{s}, g_{2}^{a} \cdot\left(h_{1}^{I_{1}} \cdots h_{k}^{I_{k}} \cdot g_{3}\right)^{r}\right)} \\
& =\frac{1}{e\left(g, g_{2}\right)^{s a}} \\
& =\frac{1}{e\left(g_{1}, g_{2}\right)^{s}}
\end{aligned}
$$

\subsection{Security}

We show that our HIBOOE scheme is selective identity secure (IND-sID-CPA) under the Decisional Bilinear Diffie-Hellman Inversion assumption. As mentioned in Section 2.3, we use a slightly weaker assumption called the Weak DBDHI*.

Theorem 3.1 Let $\mathbb{G}$ be a bilinear group of prime order $p$. Suppose the $(t, \epsilon, \ell)-w D B D H I *$ assumption holds in $\mathbb{G}$. Then the previously defined $\ell$-HIBOOE system is $\left(t^{\prime}, q_{s}, \epsilon\right)$ selective identity, chosen plaintext (IND-sID-CPA) secure for arbitrary $\ell, q_{s}$, and $t^{\prime}<t-\Theta\left(\tau \ell q_{s}\right)$, where $\tau$ is the maximum time for an exponention in $\mathbb{G}$.

Proof Sketch. Suppose $\mathcal{A}$ has advantage $\epsilon$ in attacking the $\ell$-HIBOOE system. Using $\mathcal{A}$, we build an algorithm $\mathcal{B}$ that solve the $\ell$-wDBDHI* problem in $\mathbb{G}$.

For a generator $g \in \mathbb{G}$ and $a \in \mathbb{Z}_{p}^{*}$ let $y_{i}=$ $g^{\left(a^{i}\right)}$ in $\mathbb{G}$. Algorithm $\mathcal{B}$ is given as input a random tuple $\left(g, h, y_{1}, \cdots, y_{\ell}, y_{\ell+2}, \cdots, y_{2 \ell}, T\right)$ that is either sampled from $\mathcal{P}_{w B D H I^{*}}\left(\right.$ where $\left.T=e(g, h)^{\left(a^{\ell+1}\right)}\right)$ or from $\mathcal{R}_{w B D H I^{*}}$ (where $T$ is uniform and independent in $\mathbb{G}_{1}^{*}$ ). Algorithm $\mathcal{B}$ 's goal is to output 1 when the input tuple is sampled from $\mathcal{P}_{w B D H I^{*}}$ and 0 otherwise. We do not give the proof in detail here, due to the limitation of the length of the paper. Contact the authors if needed.

Chosen Ciphertext Security. Canetti [5] provided a general method of building an IND-sID-CCA secure $\ell$-HIBE from an IND-sID-CPA secure $(\ell+1)$-HIBE. A more efficient construction is given by Boneh and Katz [4]. Applying these methods to our HIBOOE construction results in IND-sID-CCA secure $\ell$-HIBOOE for arbitrary $\ell$. 


\section{Comparison}

In a conventional HIBE, some components of ciphertext could also be pre-computed (naturally split an encryption into online/offline phases), but it is inefficient. Our HIBOOE scheme is much more efficient. We provide a comparison of computational cost in Table 1. Let "E" denote the exponentiation in $\mathbb{G}$, " $M E$ " denote the multiexponentiation in $\mathbb{G}$, " $M$ " denote the modular computation. It's clear that the algorithm " $M$ " is much faster than " $E$ ". We also assume that the message is encrypted under the public key $I D=\left(I_{1}, \cdots, I_{\ell}\right) \in\left(\mathbb{Z}_{p}^{*}\right)^{\ell}$.

\begin{tabular}{|c|c|c|}
\hline \hline Scheme & BBG-HIBE & Our HIBOOE \\
\hline Offline phase & $(\ell+3) \mathrm{E}$ & $\ell \mathrm{ME}+3 \mathrm{E}$ \\
\hline Store in offline & $\ell+3$ & $2 \ell+4$ \\
\hline Online phase & $\ell \mathrm{E}$ & $2 \mathrm{M}$ \\
\hline \hline
\end{tabular}

Table 1. This table presents a comparison of the related HIBE schemes under the IND-sIDCPA secure model. It shows that the online phase of our scheme is much more efficient than the conventional scheme.

\section{Conclusion}

In this paper, we extended the notion of Identity-based Online/offline Encryption to Hierarchical Identity-Based Online/Offline Encryption (HIBOOE) and proposed an efficient scheme, which is useful for devices with limited computational power. Our HIBOOE construction from BBGHIBE [2] is provably secure without random oracles. In the encryption phase, the offline phase encryption can be run without the message to be encrypted and the public key (or ID) of a recipient and the online phase encryption is extremely efficient with only two modular computations.

\section{Acknowledgement}

This work is supported by National Natural Science Foundation of China (60502047), Education Bureau of Fujian Province (JA07030), Natural Science Foundation of Fujian Province (2008J0014) and Science and Technology of Fujian Province (2006F5036).

\section{References}

[1] D. Boneh and X. Boyen. Efficient selective-id secure identity based encryption without random oracles.
In Advances in Cryptology-Eurocrypt'04,Vol.3027 of LNCS, pages 223-238. Springer-Verlag,2004.

[2] D. Boneh, X. Boyen, and E. J. Goh. Hierarchical Identity Based Encryption with Constant Size Ciphertext. In Cramer [16], pages 440-456. Full version available at Cryptology ePrint Archive, Report 2005/015.

[3] D. Boneh and M. Franklin. Identity-based encryption from the Weil pairing. In Joe Kilian, editor, Advances in Cryptology-CRYPTO 2001, volume 2139 of LNCS, pages 213-229. Springer-Verlag, 2001.

[4] D. Boneh and J. Katz. Improved efficiency for CCAsecure cryptosystems built using identity based encryption. In Proceedings of RSA-CT'05, LNCS 3376, pp. 87-103, 2005.

[5] R. Canetti, S. Halevi, and J. Katz. Chosen-ciphertext security from identity-based encryption. In C. Cachin and J. Camenisch, editors, Proceedings of Eurocrypt 2004, volume 3027 of LNCS, pages 207-222. Springer, 2004.

[6] C. Gentry. Practical Identity-Based Encryption Without Random Oracles. In Advance of Eurocrypt'06, vol.4004 of LNCS, pages 445-464. Springer-Verlag, 2006.

[7] F. Guo, Y. Mu, and Z. Chen. Identity-Based Online/Offline Encryption. Proceedings of FC 2008, LNCS 5143, pp. 247-261. Springer-Verlag Berlin Heidelberg 2008.

[8] C. Gentry and A. Silverberg. Hierarchical ID-Based Cryptography. In Proc. ASIACRYPT 2002, volume 2501 of Lecture Notes in Computer Science, pages 548566. Springer-Verlag, 2002.

[9] J. Horwitz and B. Lynn. Towards hierarchical identity-based encryption. In Advances in CryptologyEUROCRYPT 2002, Lecture Notes in Computer Science, pages 466-481. Springer-Verlag, 2002.

[10] A. Shamir. Identity-based cryptosystems and signature schemes. In Advances in Cryptology-CRYPTO 1984, volume 196 of LNCS, pages 47-53. SpringerVerlag, 1984.

[11] T. H. Yuen and V. K. Wei. Constant-Size Hierarchical Identity-Based Signature/ Signcryption without Random Oracles. Cryptology ePrint Archive, Report 2005/412, 2005.

[12] B.Waters: Efficient Identity-Based Encryption without Random Oracles. In advance in CryptologyEurocrypt'05, vol.3494 of LNCS, pages 114-127. Springer-Verlag, 2005. 